\title{
A Standardized Protocol for Cone-Beam Computed Tomography of the Hand and Wrist in Rheumatoid Airthritis
}

\author{
DOI: $10.17691 / \mathrm{stm} 2015.7 .4 .18$
}

Received May 25, 2015

D.V. Makarova, MD, PhD, Assistant, Department of Radiology';

K.V. Kushnyr, MD, PhD, Chief of Radiology Center ${ }^{2}$

${ }^{1}$ Moscow State University of Medicine and Dentistry named after A.I. Evdokimov, 20/1 Delegatskaya St., Moscow, 127473, Russian Federation;

${ }^{2}$ Main Clinical Hospital, Ministry of Internal Affairs of the Russian Federation, 35 Narodnogo opolcheniya St., Moscow, 123060, Russian Federation

The aim of the investigation was to develop a standardized protocol for description of the results of cone-beam computed tomography (CBCT) of the hand and wrist in rheumatoid arthritis.

Materials and Methods. The results of 112 hand and wrist CBCT examinations $(n=56)$ were analyzed in patients aged from 36 to 67 with medium-active seropositive rheumatoid arthritis in anamnesis. The hand and wrist CBCT was carried out using a NewTom 5G cone-beam computed unit (QR s.r.l., Italy) having a $200 \times 250 \mathrm{~mm}$ flat-panel detector, $180 \times 160 \mathrm{~mm}$ field of view (maximum), and a $360^{\circ}$ gantry rotation around the region of interest. The hand and wrist scanning was carried out from the distal metaphysis of the forearm bones to the nail ends of the distal phalanges of the fingers. Scan mode - patient scan (exposure time $-3.6 \mathrm{~s}$, X-ray tube boosting voltage - $110 \mathrm{~kW}$, current 0.6-0.8 mA), scan pattern — regular scan, scan time - $18 \mathrm{~s}$. The CBCT examinations of the wrists and hands were performed using specialpurpose setups allowing complete coverage of the region of interest.

Results. A standardized protocol was designed for the description of hand and wrist cone-beam examination that documents all the major changes resulting from rheumatoid arthritis: soft tissue thickening, erosions and cystic-like changes, osteoporosis, narrowing of the joint spaces, incomplete dislocations, bone deformities and areas of osteolysis. Significant changes are recorded in point form, according to the Sharp or SENS methods. Application of the standardized protocol allows unification of the structuring of the examination description, and facilitates X-ray assessment of changes in the hand and wrist during CBCT in patients with rheumatoid arthritis.

Key words: cone-beam computed tomography; standardized protocol; rheumatoid arthritis; osseous structure.

The leading clinical evidence of rheumatoid arthritis (RA) is the different intensity of articular syndrome, combined with acute polyarthritis with predominant involvement of the hand and foot joints, as well as recurrent bursitis and tenosynovitis of the wrist joints [14]. The advantages of magnetic resonance tomography (MRT) and ultrasound investigations (USI) in the diagnosis of the inflammatory process and destructive changes to the joints in patients with RA are detailed in the recently published results of two studies [3, 5]. In 2013, the recommendations of the European League Against Rheumatism (EULAR) were presented; according to these recommendations, conventional radiology should be used as the first stage of diagnosis of hand and foot damage in patients with RA. If no changes are observed with this method, USI and/or MRT are indicated [5].

Frequently, however, in everyday clinical practice, Xray diagnostics of $R A$, and specifically, assessment of the severity of joint disease of the hands and feet is based only on standardized roentgenograms. According to domestic recommendations, in the diagnosis statement of such patients it is necessary to indicate the clinical features of the disease (arthritis, extra-articular lesions), variant (by the presence of rheumatoid factor), course features, level of activity (according to clinical and laboratory data), extent of capacity disturbance, and the current radiological stage. Initially, this radiological stage is assessed on the basis of radiologic abnormalities in the small joints of the hand and at the distal ends of the foot. For this purpose, Steinbrocker's radiographic grading of RA (1949) is currently the most widely used. Based on this classification, four disease states can be distinguished [2, 4].

According to Larsen, for assessment of the progression of joint abnormality in RA, the Sharp and SENS methods, the presence and degree of erosion of the articular surface and the extent of joint space narrowing are of relevance [4, 6-8]. Unfortunately, conventional radiology by no means always allows a detailed assessment of the changes in osseous structure in RA [3, 5, 9]. According to certain authors [10], multi-slice computed tomography (CT) is the most informative method for identifying specific radiographic abnormalities, however, its application in clinical practice

For contacts: Makarova Darya Valer'evna, e-mail: mdvmail@mail.ru 
is limited by many factors, in particular the relatively high radiation exposure required.

Nowadays, as a result of the development of up-todate special-purpose cone-beam computed units, it is possible to use this technique to examine distal segments of the upper and lower limbs. Cone-beam computed tomography (CBCT) imaging is based on scanning the region of interest using a pulsed X-ray beam collimated so that the radiation occurs in the form of a cone. The tissue-attenuated $\mathrm{X}$-ray radiation then reaches at a flat-panel detector. The use of cone-beam technology means that a single turn of the $\mathrm{X}$-ray tube around the target generates a primary image ready for further postprocessing. Another important advantage of CBCT vs. multi-slice CT is the potential for a significant reduction in the exposure dose, due to the short duration of the direct X-ray irradiation and the high sensitivity of the flatpanel detector. These capabilities and advantages mean that $\mathrm{CBCT}$ provides an expedient alternative to multislice CT for the examination of the hands of RA patients $[9,11-13]$.

The available literature indicates that only a few publications have addressed the applicability of CBCT for RA patients [11]. Moreover, there is no standardized protocol for the description of the results of such examinations.

The aim of the investigation was to develop a standardized protocol for the description of the results of hand and wrist cone-beam computed tomography of rheumatoid arthritis.

Materials and Methods. The results of 112 hand and wrist CBCT examinations $(n=56)$ were analyzed in patients aged from 36 to 67 with medium-active seropositive rheumatoid arthritis in anamnesis. All the examinations were conducted in the Radiological Center of the Main Clinical Hospital, Ministry of Internal Affairs of Russia. Of the patients there were 42 women and 14 men. The duration of the disease varied from 1.5 to 30 years. The study group excluded persons with immediate-early ( $<6$ months) and early (from 6 months to 1 year) stages of the disease. The extensive stage of RA (duration of the disease $>1$ year, with typical symptoms) was detected in $26.8 \%(n=15)$ of those examined and the late stage (>2 years, with marked destructive changes and complications) - in $73.2 \% \quad(n=41)$. These patients were divided according to the RA activity (using the DAS28 scale) into two groups: FC II — medium (3.2$5.1)-25$ persons $(44.6 \%)$, and FC III - high $(>5.1)-$ 31 people $(55.4 \%)$.

The study was carried out in accordance with the Declaration of Helsinki (adopted in June 1964 (Helsinki, Finland) and revised in October 2000 (Edinburgh, Scotland)) and approved by the local ethics committee.

All patients gave written, informed consent for CBCT examinations and the scientific analysis of their data. CBCT of their hands and wrists was carried out using a cone-beam computed unit (NewTom 5G, QR s.r.l.,
Italy) having the following technical characteristics: $200 \times 250 \mathrm{~mm}$ flat-panel detector size, $180 \times 160 \mathrm{~mm}$ maximum field of view, and a $360^{\circ}$ gantry rotation around the region of interest. The hand and wrist scanning was performed from the distal metaphysis of the forearm bones to the nail ends of the distal finger phalanges. Settings used: scan mode - patient scan (exposure time $-3.6 \mathrm{~s}$, X-ray tube boosting voltage $110 \mathrm{~kW}$, current $-0.6-0.8 \mathrm{~mA}$ ), scan pattern - regular scan, scan time $-18 \mathrm{~s}$. The CBCT hand and wrist examinations were carried out in special-purpose positioning setups allowing for full coverage of the region of interest.

For determining the severity of articular syndrome we used a general method, according to the Steinbrocker's radiographic grading (1949), for assessment of the changes in the joints caused by RA as part of the postprocessing of the hand and wrist $\mathrm{CBCT}$ tomograms.

For this purpose the following presence/absence of RA radiological signs was noted:

soft tissue diffusive periarticular thickening and hardening;

osteoporosis (circumarticular and generalized);

cystic-like changes;

joint space narrowing;

articular surface erosion (marginal, compressive and at the ligament attachment points) and areas of osteolysis;

bone ankylosis;

bone deformities;

complete dislocations and incomplete dislocations.

Subsequently, by analogy with standardized roentgenograms, quantitative methods were used for assessment of the RA changes in the joints: Sharp, as modified by van der Heijde (1989) and SENS (Simple Erosion Narrowing Score).

The Sharp method, as modified by van der Heijde, was used for assessment of the bone articular surface condition in 16 joints, and for erosion changes and the degree of joint-space narrowing in 15 joints, of each hand. The number of erosions and the severity of narrowing were scored. The total number of score points for both hands therefore covered a range from 0 to 280 .

The SENS method was used for simple calculation of the erosion in 32 joints and/or the joint-space narrowing in 30 hand joints. Here, the total number of score points for both hands was therefore from 0 to 62 .

Results and Discussion. A standardized protocol for the description of hand and wrist CBCT was developed and tabulated (See the Table) for the purpose of data unification as well as for process optimization of the general or quantitative count of the detected changes, according to any of the usual methods for RA jointchange assessment.

Information about hand and wrist changes in RA obtained in the course of post-processing using 
Sample standardized protocol for description of cone-beam computed tomography of the left hand and wrist

\begin{tabular}{|c|c|c|c|c|c|c|}
\hline \multicolumn{2}{|l|}{ Joints } & \multicolumn{2}{|c|}{ Soft tissues } & & $\begin{array}{l}\text { ncomplete } \\
\text { islocations }\end{array}$ & Ankylosis \\
\hline \multicolumn{2}{|l|}{ Wrist } & \multicolumn{2}{|l|}{+} & $2-3$ & - & - \\
\hline \multicolumn{2}{|c|}{ Magnum scaphalunate ligament } & \multicolumn{2}{|l|}{+} & 3 & - & - \\
\hline \multicolumn{2}{|c|}{ Navicular trapezoid } & \multicolumn{2}{|l|}{+} & & - & - \\
\hline \multicolumn{2}{|l|}{ Carpometacarpal } & \multicolumn{2}{|l|}{+} & $3-3-3-3-3$ & - & - \\
\hline \multicolumn{2}{|l|}{ Metacarpophalangeal } & \multicolumn{2}{|l|}{+} & $2-3-2-2-2$ & - & - \\
\hline \multicolumn{2}{|l|}{ Proximal interphalangeal } & \multicolumn{2}{|l|}{-} & $1-1-1-1-0$ & - & - \\
\hline \multicolumn{2}{|l|}{ Distal interphalangeal } & - & \multicolumn{2}{|c|}{$0-0-0-0$} & - & - \\
\hline Bones & Porosis & Cysts & Erosions & Osteolysis & & deformity \\
\hline Distal ulnar epiphysis & + & + & 2 & - & & - \\
\hline Radial epiphysis & + & + & 3 & - & & - \\
\hline Navicular & + & + & 3 & - & & - \\
\hline Lunate & + & + & 3 & - & & - \\
\hline Triquetral & + & + & 3 & - & & - \\
\hline Postulnar & + & + & 2 & - & & - \\
\hline Trapezium & + & - & 2 & - & & - \\
\hline Trapezoid & + & - & 1 & - & & - \\
\hline Capitate & + & + & 2 & - & & - \\
\hline Uncinatum & + & + & 3 & - & & - \\
\hline Metacarpal bones & + & III-V & $1-3-2-2-1$ & - & & - \\
\hline Proximal phalanx & + & I, III, V & $2-2-1-1-1$ & - & & - \\
\hline Middle phalanx & - & - & 0 & - & & - \\
\hline Nail bones & - & - & 0 & - & & - \\
\hline
\end{tabular}

sequential analysis of cross-scans and multiplanar reconstructions is recorded in the standardized protocol (erosion and narrowing are marked with scores (from 0 to 5), while the remainder are marked with '+' or '-' signs). Changes of metacarpal bones, distal finger phalanges, and the carpometacarpal, metacarpophalangeal and interphalangeal joints are marked in the table with a hyphen and denoted according to their sequence numbers.

Subject to clinicians' needs, standardized protocol data can be used not only to indicate the radiological stage of the pathologic process but also to calculate comparable scores both in respect of selected general or quantitative methods of assessing RA-caused changes in the joint, by summing the scores of the items required. The data obtained from $C B C T$ hand and wrist scans of RA patients and recorded according to this standardized protocol are more readily visualized, and this substantially simplifies comparison of cone beam studies involving sequential scans during interactive assessment of movement responses.

The following clinical observation is a practical example of the use of the standardized protocol.

Patient L.: year of birth 1967, diagnosis: "seropositive rheumatoid arthritis (MO5.8), advanced stage, activity -
FC II (DAS28=3.2), erosive (radiological stage II), with systemic symptoms (rheumatoid nodules, digital arteritis), cyclic citrullinated peptide antibodies - negative". Has been suffering from the disease for 7 years.

The patient underwent hand and wrist CBCT for both limbs at the treatment planning stage in order to enable outcome monitoring. The data obtained in the course of the cone-beam image post-processing (Figures 1 and 2) were recorded using the standardized description protocols.

The standardized protocol for the patient's left hand CBCT data (See the Table) is drawn up in dynamics. The scoring before treatment was 87 points according to the Sharp method, as modified by van der Heijde, and 28 points according to the SENS method. The comparable cone-beam examination results obtained after the treatment showed scores of 92 and 30 points for the respective Sharp and SENS methods, providing evidence of the extent of treatment success.

Conclusion. Use of this standardized protocol for hand and wrist cone-beam computed tomography in rheumatoid arthritis allows for unification and structuring of the examination description and accelerates its completion. Its application substantially facilitates the scoring process according to both of the selected methods. Moreover, use of the suggested protocol 


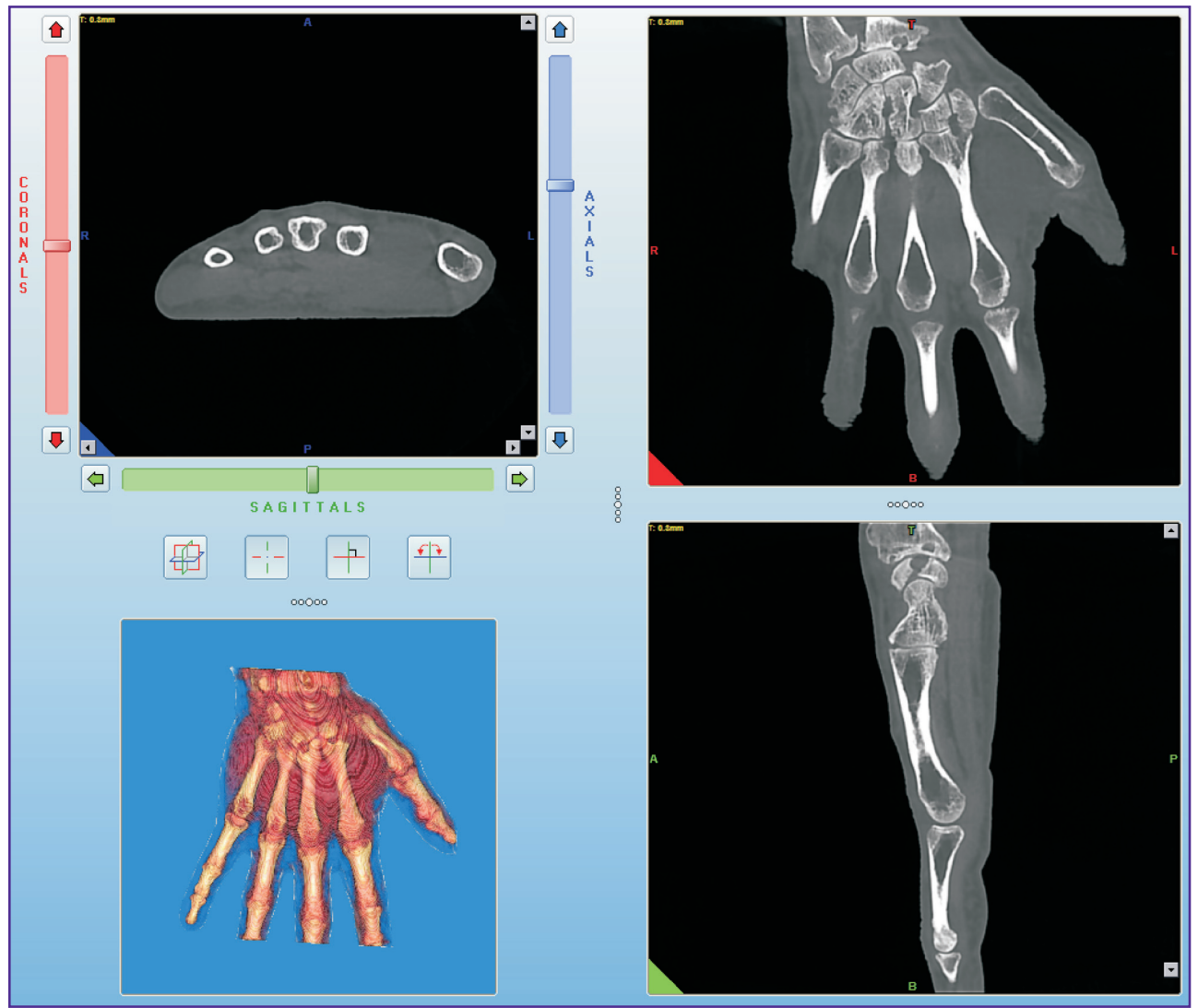

Figure 1. Multiplanar cone-beam computed tomograms of patient L. - left hand and wrist. In the setting of diffuse osteoporosis, multiple marginal usuras and cystic cavities of the carpal bones, and distal epiphysis of the radial and ulnar bones are visualized. At this level, the articular surfaces are flattened, the subchondral plates are scleroid, and marginal osteophytes are detected mostly in the planes of the distal ends of the radial and ulnar bones. Severity III narrowing of the radial-navicular, wrist and carpometacarpal joints is detected, while the metacarpophalangeal and proximal interphalangeal joints indicate severity II
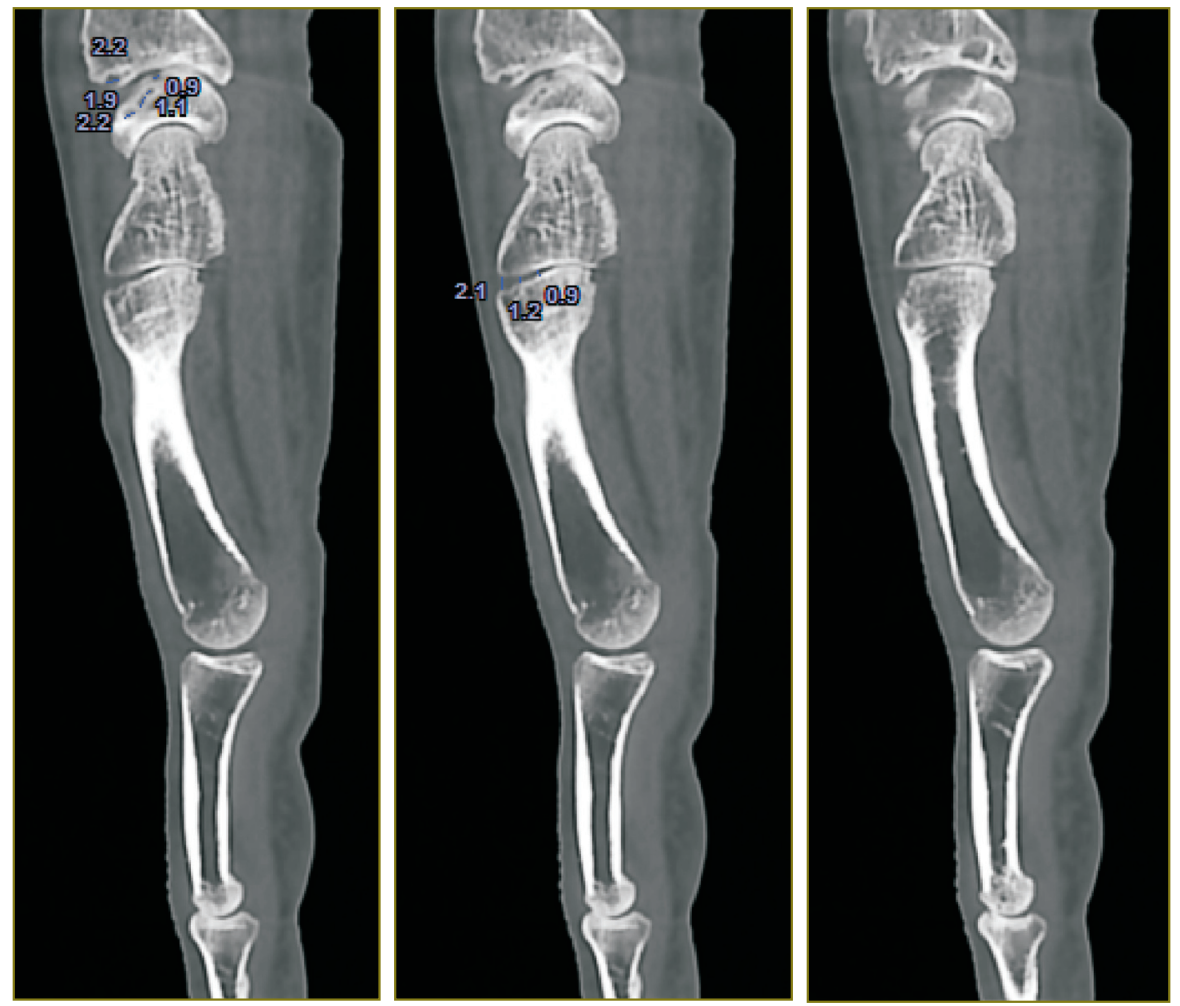

Figure 2. Cone-beam computed tomograms of patient L. - left hand in sagittal plane. In the setting of diffuse osteoporosis, at the level of the subchondral sections of the radial and lunate bones, multiple marginal usuras and cystic cavities from $0.9 \mathrm{~mm}$ upwards are visible. At this scan level, all the joint spaces appear unevenly narrowed, while the articular surfaces are flattened and scleroid 
simplifies interpretation of the results obtained, including where CBCT is used to assess the hand in dynamics, for determining the response to the treatment being provided. Standardization of the descriptions of the detected changes allows for the application of the highresolution $\mathrm{CBCT}$ technique in everyday rheumatologic practice.

Study Funding and Conflicts of Interest. The study was not financed from any sources, and there are no conflicts associated with this study.

\section{References}

1. Myasoedova S.E., Lesnyak O.M., Men'shikova L.V., Bol'shakova T.Yu., GalushkoE.A.,ProtopopovaR.N., Erdes Sh.F., Chernykh T.M., Vinogradova I.B., Bazorkina D.I. Prevalence of rheumatoid arthritis in Russia (according to epidemiological researches). Terapevticheskii arkhiv 2010; 82(5): 9-14.

2. Useinov R.K., Iskova I.A., Zakharova S.N., Klyaritskaya I.L. Experience in the treatment of rheumatoid arthritis. New technologies (case report). Kryms'kyj terapevtychnyj zhurnal 2012; 1: 127-129.

3. Osipyants R.A., Karateev D.E., Panasyuk E.Yu., Lukina G.V., Smirnov A.V., Glukhova S.I., Aleksandrova E.N., Volkov A.V., Nasonov E.L. Evaluation of the structural changes of the hand joints and the rates of rheumatoid arthritis progression according to ultrasound data. Nauchno-prakticheskaya revmatologiya 2013; 51(2): 132-137.

4. Smirnov A.V. Atlas rentgenologicheskoy diagnostiki revmatoidnogo artrita [Atlas of rheumatoid arthritis diagnostic radiology]. Moscow: IMA-PRESS; 2009.

5. Colebatch A.N., Edwards C.J., Østergaard M., van der Heijde D., Balint P.V., D'Agostino M.A., Forslind K., Grassi W., Haavardsholm E.A., Haugeberg G., Jurik A.G.,
Landewé R.B., Naredo E., O’Connor P.J., Ostendorf B., Potocki K., Schmidt W.A., Smolen J.S., Sokolovic S., Watt I., Conaghan P.G. EULAR Recommendations for the use of imaging of the joints in the clinical management of rheumatoid arthritis. Ann Rheum Dis 2013; 72(6): 804-814. URL: http:// dx.doi.org/10.1136/annrheumdis-2012-203158.

6. Sommer O.J., Kladosek A., Weiler V., Czembirek H., Boeck M., Stiskal M. Rheumatoid arthritis: a practical guide to state-of-the-art imaging, image interpretation and clinical implications. Radiographics 2005; 125(2): 381-389, http:// dx.doi.org/10.1148/rg.252045111.

7. van der Heijde D.M. How to read radiographs according to the Sharp/van der Heijde method. J Rhematol 1999; 26(3): 743-745.

8. van der Heijde D., Dankert T., Nieman F., Rau R., Boers M. Reliability and sensitivity to change of a simplification of the Sharp/van der Heijde radiological assessment in rheumatoid arthritis. Rheumatology (Oxford) 1999; 38(10): 941-947, http:// dx.doi.org/10.1093/rheumatology/38.10.941.

9. Makarova D.V. Cone-beam computed tomography opportunities in bone and joint studies. Vrach-aspirant 2013; 61(6.2): 282-289.

10. Mudge C.S., Yoo D.C., Noto R.B. Rheumatoid arthritis demonstrated on PET/CT. Med Health R I 2012; 95(2): 55.

11. Aurell $Y$., Malac M., Forslind K. Work in progress: cone beam computed tomography (CBCT or extremity $\mathrm{CT}$ ) - a new tool for evaluation of erosions in patients with rheumatoid arthritis. ESSR 2014, http://dx.doi.org/10.1594/essr2014/P0028.

12. Koh K.J., Kim K.A. Utility of the computed tomography indices on cone beam computed tomography images in the diagnosis of osteoporosis in women. Imag Sci Dent 2011; 41(3): 101-106, http://dx.doi.org/10.5624/isd.2011.41.3.101.

13. Kokkonen H. Development and evaluation of delayed CT arthrography of cartilage. Acad. Diss. 2012. 